\title{
Characterization of Polymers and Catalysts Using Scanning Transmission Electron Microscopy (STEM) in a Field Emission SEM
}

\author{
G.M. Brown and A.D. Westwood
}

Exxon Mobil Chemical Company, Baytown Polymers Center, 5200 Bayway Drive, Baytown, Texas, 77520-2101

High resolution, analytical transmission electron microscopy of polymeric and catalyst systems is now routinely performed in FE-SEM. The SEM/STEM consists of a Hitachi S-4300 FE-SEM equipped with transmission electron and EDS detectors. A probe size of $8 \mathrm{~nm}$ FWHM and $20 \mathrm{~nm}$ FWTM is routinely achieved at $30 \mathrm{kV}$ accelerating voltage with probe currents as high as $13 \mathrm{nA}$. Taking into account beam broadening effects, our analytical spatial resolution is $\sim 30 \mathrm{~nm}$ (probe 20 $\mathrm{nm}$ FWTM) for a $100 \mathrm{~nm}$-thick elastomeric sample of $0.92 \mathrm{gcm}^{-3}$ density. Brightfield, transmission electron imaging is achieved using a Hitachi scintillator-type TE detector. Typical sections $(\leq 100$ $\mathrm{nm}$-thick) are prepared by ultramicrotomy or focussed ion beam (FIB) milling and supported on continuous or lacey carbon film grids. In STEM mode, the spatial x-ray analytical resolution of the FE-SEM with EDS is surpassed only by a FE-TEM(STEM) mode or a dedicated STEM.

Polyolefin plastics, elastomers and zeolitic materials are typically very beam sensitive. Attempts to perform bulk analysis of the catalysts and polymers by SEM or FE-SEM are generally futile and high accelerating voltage TEM analyses often result in the scissioning and volatilization of polymers and amorphization and possible chemical changes in zeolites. Benefits of using the SEM/STEM for EDS mapping include: (a) little or no beam damage is observed in polymers, catalysts or zeolites; (b) absence of electrostatic charging by the sample facilitates imaging and $\mathrm{x}$-ray microanalysis; (c) good image contrast often eliminates the need for heavy metal staining (i.e. $\mathrm{RuO}_{4}$ and $\mathrm{OsO}_{4}$ ) of elastomers (imaging the crystalline morphologies of many polymers still requires staining); and (d) very low magnification imaging of larger structure such as filler aggregates, large polymeric domains, etc.

This method has been applied to a number of material systems. These include imaging and elemental mapping of the homogeneity and distribution of fillers and curatives in elastomers FIG. 1., and the lamellar morphology of $\mathrm{RuO}_{4}$-stained semi-crystalline polymers FIG. 2. and 3. Catalyst-related applications include the morphology of silica aerogels and zeolites as catalyst supports and the distribution of catalyst metals, poisons and contaminates on these supports.

FIG. 1. Brightfield SEM/STEM image and EDS spectral maps of additives and fillers in cured butyl rubber. In the absence of EDS, dark spots such as those seen here are often assumed to be $\mathrm{ZnO}$ particles. In fact, these particles have diverse compositions. The large particle (left center of transmitted electron image) contains all detectable additives whereas several $\mathrm{ZnO}$ particles were also seen. FIG. 2. illustrates the large-scale domain morphology of a $\mathrm{RuO}_{4}$-stained blend of two semicrystalline polyolefins. FIG. 3. A fan-shaped lamellar bundle in a semi-crystalline polymer. FIG. 4. FIB cross-section (100 nm-thick) through a zeolitic membrane shows a continuous siliceous zeolitic layer overlying the porous $\alpha$-alumina support layer. The black zone (at right) is a protective platinum layer applied prior to FIB milling. FIG. 5. STEM image and EDS spectrum of chromium acetate (catalyst precursor) on silica. $[1,2]$

[1] Presented at 161 st Rubber Division Meeting, ACS, Savannah, GA, April 2002 and pending publication in Rubber Chemistry and Technology, Rubber Division of ACS.

[2] Thanks to Drs. P. Brant, A.N. Speca and A.J. Bons for samples and Ms. J.E. Cox for cryoultramicrotomy. 

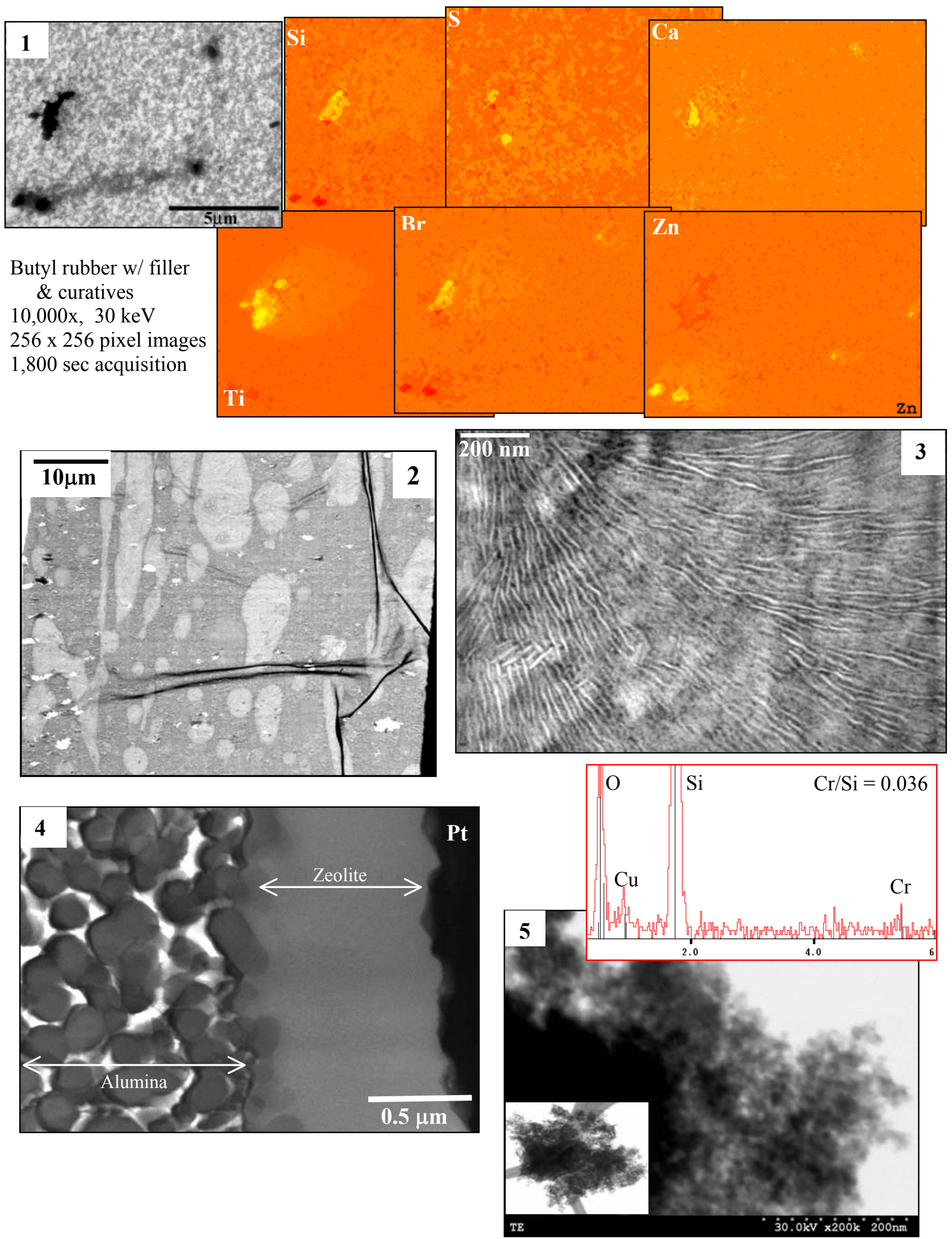

Copyright 2003 Exxon Mobil Corporation. All rights reserved. 